\title{
A SURVEY OF THE DETERMINANTS OF EFFECTIVE E-LEARNING EDUCATION IN NIGERIAN SECONDARY SCHOOLS: A WAY OF IMPROVING ECONOMICS LEARNING AND SOCIAL DEVELOPMENT IN POST COVID-19
}

\author{
JOVITA CHINELO EJIMONYE ${ }^{1}$, NJIDEKA DORATHY ENEOGU ${ }^{1 *} \&$ JOY CHIKODILI OMALIKO ${ }^{2}$ \\ ${ }^{1}$ Department of Social Science Education, University of Nigeria, Nsukka \\ ${ }^{2}$ Department of Sociology and Anthropology, University of Nigeria, Nsukka
}

\begin{abstract}
E-learning education has been a concern in education system in developing countries including Nigeria after Covid-19 pandemic. It is obvious that the state of e-learning education in Nigerian Secondary Schools in not encouraging and this called for more investigation. The main purpose of this study is to investigate the determinants of effective use of elearning education in Nigerian Secondary Schools for a better Economics instruction and social development in post Covid-19. The study explores factors such as availability of technological facilities, teachers' demography and teachers training on e-learning education using data from National Bureau of Statistics (NBS), 2018. The study employs both descriptive and regression approach in the analysis. The findings, among other things, revealed that the major determinants to effective e-learning education are availability of technological facilities and funding of Nigerian Secondary Schools. This may affect Economics learning and social development in post Covid-19 pandemic. The study recommended that there is need for government and stakeholders of Nigerian Secondary Schools to increase funding in procuring technological facilities for schools. Teachers' in-service training is important in order to acquire the necessary knowledge and skills needed for effective learning of Economics in Secondary Schools.
\end{abstract}

KEYWORDS: E-learning, Education, Economics, Secondary schools, Covid-19

Received: Mar 27, 2021; Accepted: Apr 17, 2021; Published: Apr 24, 2021; Paper Id.: IJMPERDJUN202120

\section{INTRODUCTION}

Corona virus Disease 2019 (Covid-19) has affected the whole sectors of economy including education sector. It is a global problem which led to many countries to lockdown their economic activities. Covid-19 started in December 2019 in Wuhan in China. It was declared as pandemic by the world Health Organization (WHO) on $11^{\text {th }}$ March, 2020 due to the rate of its transmission (Dos Santos, 2020). It is one of the public health challenges in the whole world both developed and developing countries. As it is now, the world total cases is 54, 328, 752 in which Asia is 15,002,645 while Europe is 13, 733,675 (WHO, 2020). Presently, Nigeria total cases is 66, 228 (NCDC, 2020). Nigeria Centre for Disease control (NCDC) reported that the training of the rapid response teams across the 36 states in Nigeria was completed in December 2019 and they are ready to respond to any emergency by $28^{\text {th }}$ January, 2020 (Ihekweazu, 2020). The first case of Corona virus in Nigeria was discovered on $27^{\text {th }}$ February, 2020 and it was imported from Italy by an Italian. The index visited many places and had contact with people before testing positive for Covid-19 (Anzat, Aminu, Kolo, Akinyele, Ogundairo \& Danjibo, 2020). Covid-19 pandemic made some Nigerians that are outside the country to come back home and some of them came back with positive for Covid-19. This increased the spread of the disease across the states through inter-state travel. This raised alarm 
and serious concern to Nigerians and distort the whole economic activities and education system.

Schools and learning were shut down and everywhere was lockdown from March to September 2020 to avoid further spreading of the disease. Students were at their various homes doing various chaos household, eating and sleeping without any formal education. Many of them forgot what they have learnt and the most affected group were the current classes. In support of the above, the United Nations revealed that the covid-19 has interrupted education system worldwide and it affected 1.6 billion learners in more than 190 countries and all continents (UN, 2020). During the period of lockdown, UNESCO monitoring reported that about 191 countries implemented nationwide closures which affected 98.4 percent of the world's student population. Preventive measures such as social distancing, self-isolation, washing of hands with soap and hand sanitizers were initiated and these prompted the widespread closure of primary, secondary and tertiary institutions in over 100 countries (Adelakun, 2020). According to UNICEF, the shutdown of Nigerian schools due to covid-19 pandemic made more than 50 million children to stay at home without formal education. The aftermath of this pandemic may increase students dropout especially in secondary schools and this will not be visible now rather it will manifest in the future because the learning loss during that period of lock down reduced the learning opportunities in acquiring more knowledge and skills and the gap may not be recovered. The pandemic really affected vulnerable children, youth, poor, refuges, and people with disabilities among others to continue their learning.

Nigerian secondary schools during the period of covid-19 pandemic lockdown were in a state of dilemma on how they will continue learning irrespective of the disruption of education system. In order to mitigate the risk of covid-19 pandemic, the federal Ministry of Education ordered to use online and offline platforms, televisions, radio and take-home materials to heighten learning (UNICEF, 2020). There were innovative approaches for learning continuity. Learning was going on in some of the social media devices such as radio, television, WhatsApp, Zoom and take-home packages. Not all the secondary schools were fortunate to meet up and join the online learning and the reason is what this study sought to find out. Some schools were able to embark on innovative learning approaches by educating their teachers through online on how to deliver their instructions with technological devices while some schools found it difficult to meet up the challenges of this great covid-19 pandemic. The reason could be inadequate technological facilities, lack of knowledge and skills to operate technological devices, instability in power supply and inadequate funding. All these could be hindrances for effective e-learning education especially in Economics and could deprive Economics teachers from using innovative teaching pedagogies in teaching and learning. Covid-19 pandemic has escalated the need for technological knowledge and skills for a better future in Education. E-learning has come to stay, and all the sectors of the economy are embracing it including the education sector.

E-learning is the process of using technological devices in delivery instructions to the learners. According to Arghya, Pradip and Shilpee (2020), e-learning is defined as computer based such as digital videos, tables, projector, operating system for learning process and interaction of students and teachers. Basak, Wotto and Belanger (2017) defined e-learning as a type of distance learning that is capable to help in achieving the education objective for all. It provides an opportunity for teachers and learners that are far away to interact. This implies that it enhances interactivity and connectivity in teaching and learning. Advantages of e-learning in educational system are highly encouraging in such a way that it modernizes the teaching techniques while the traditional method of teaching is gradually facing away. The adoption of e-learning education in West Africa is still at the beginning stage (Opoku and Adu, 2016) that is why some schools in Nigeria are still finding it difficult to implement it .E-learning education has lots of advantages that help to 
overcome the challenges of the problem of Covid-19. Petit, Wharrad and Windle (2013) discovered some advantages of elearning as follows: reducing the cost of traditional classroom learning, enhancing coordination and cooperation among the learners and enables the instructors to evaluate the progress of the information delivery. In order to buttress the advantages of e-learning, Wanyaga, kamau and Gikandi (2015) found that e-learning intensifies learners' motivation, provide easy accessibility and enhances creative communication and learners thinking. Upon all these advantages, some countries in West Africa are still lagging behind in adopting and implementing e-learning education. Khasawneh, 2015 noted that some of the countries in West Africa are not yet ready to accept technology. Covid-19 pandemic has made the developing countries including Nigeria to discover the need for the adoption of e-learning in education system and yet there is a problem of implementation. Now, most of the schools have resumed. School activities are no longer in a normal state. School timetables and schedules have changed. Some states resumed with third term of 2019/2020 session while other states resume with first term of 2020/2021 session. Covid-19 aftermath affected most of the schools in such a way that some schools have started adopting e-learning in delivery instructions while teachers update themselves by acquiring more technological knowledge to face the challenges of this post Covid-19 era. Some schools that used e-learning during the lockdown taught many subjects including Economics.

Economics involves both quantitative and qualitative aspects and students need to understand the both for a better knowledge. Teaching quantitative concepts of Economics through online require more technological skills, good knowledge of software and the quality of e-learning system. Yakubu and Dasuki, 2018 commented that the quality of the e-learning system affects positively the quality of teaching in an e-learning environment. This implies that effective elearning depends on the types and quality of e-learning facilities that are available in the school. Good software can design quantitative Economics concepts in a comprehensible way so that it will be easier for the students to assimilate. Economics is a subject for everyone, and economics activities concern every individual. There is need to improve the delivery of Economics instruction through the use of effective e-learning education. Therefore, in spite of the effort made so far by the federal government in providing computers and technological facilities to Nigerian secondary schools, yet, many schools in Nigeria found it difficult to adopt e-learning education as it was manifested during Covid-19 lockdown.

The problem of this study is of the fact that even after the Covid-19 lockdown, Many Nigerian secondary schools were unable to continue their online learning because they cannot meet up with the demand of e-learning even when the federal government mandated all the schools to adopt e-learning education for continuity. Technology has come in education industry in such a way that the traditional method of teaching is facing out gradually. Developed countries have transformed their education system into technological habitant. Many sectors of economy including education are adopting online and e-learning education; yet, some secondary schools in Nigeria are facing the challenges and still maintaining the traditional way of teaching. There is need for adjustment in Nigerian secondary schools to welcome new technologies, new skills and other changes that manifested in post covid-19 era. Some teachers need in-service training to improve their knowledge in technological skills. Thus, based on this backdrop, it is necessary to determine the factors affecting the effective use of e-learning education in Nigeria secondary schools to improve Economics learning in post covid-19 era.

Theoretically, this study anchored on theory of technology organization environment (TOE). The theory was excogitated by Tornatzky and Fleischer (1990). The tenet of this theory is that effective e-learning depends on the pattern which the organizations or institutions adopt and also how they implement technological perspective and the environmental perspective. The theory further explained that these three elements show both limitations and opportunities 
for technological adoption and use. The relationship between the theory and this study is that the manner in which Nigerian secondary schools view e-learning education will determine its effectiveness. The justification of this theory to this study is that Nigerian secondary schools require experts that will help to improve operational efficiency of technological devices. In terms of organizational perspective, Nigerian secondary schools' employees should be well-educated on how to utilize new technology in delivery instructions. Principals and teachers need to work hand in hand with team spirit because team diversities enhance creativity and productivity for effective delivery. Technology organization environment theory incorporates a wide range of factors that can help in adoption of e-learning facilities in Nigerian secondary schools. Also, it is suitable for this study because secondary schools deal with physical facilities that promote learning and functions which have the factors associated technology, organization and environment that may affect the use of effective e-learning facilities.

Purpose of the study

- To identify e-learning facilities in Nigerian secondary schools.

- To identify secondary schools teachers' qualification for effective e-learning education.

- To examine secondary schools teachers' computer literacy for effective e-learning education.

\section{Research Questions}

- What are e-learning facilities in Nigerian secondary schools?

- What is secondary schools teachers' qualification for effective e-learning education?

- How many secondary schools teachers' have computer literacy for effective e-learning education?

\section{METHODOLOGY}

This study used data from the report of National Bureau of statistics (NBS) (2017, 2018 and 2019) to investigate the determinants of effective use of e-learning education in Nigerian secondary schools across the states in post convid-19 era. Descriptive statistics was used to analyse the data. With this method, states that have more technological facilities with more teachers that have technological knowledge and skills above 50\% tend to have effective e-learning education than their counterparts.

\section{RESULTS}

Research Question 1: The analysis of percentage of e-learning facilities in Nigerian secondary schools?

Table 1: Number of Schools with Facilities by State by Type in Public and Private Secondary School

\begin{tabular}{|c|l|c|c|c|c|c|c|c|c|c|}
\hline S/N & State & $\begin{array}{c}\text { No } \\
\text { Schools }\end{array}$ & $\begin{array}{c}\text { Source of } \\
\text { Power }\end{array}$ & $\mathbf{\%}$ & Computer & \% & Lab & \% & Library & \% \\
\hline $\mathbf{1}$ & Abia & 798 & 572 & $72 \%$ & 504 & $63 \%$ & 458 & $57 \%$ & 449 & $56 \%$ \\
\hline $\mathbf{2}$ & Adamawa & 747 & 317 & $42 \%$ & 239 & $32 \%$ & 140 & $19 \%$ & 150 & $20 \%$ \\
\hline $\mathbf{3}$ & $\begin{array}{l}\text { Akwa- } \\
\text { Ibom }\end{array}$ & 624 & 473 & $76 \%$ & 376 & $60 \%$ & 444 & $71 \%$ & 489 & $78 \%$ \\
\hline $\mathbf{4}$ & Anambra & 866 & 682 & $79 \%$ & 690 & $80 \%$ & 632 & $73 \%$ & 700 & $81 \%$ \\
\hline $\mathbf{5}$ & Bauchi & 910 & 287 & $32 \%$ & 238 & $26 \%$ & 104 & $11 \%$ & 119 & $13 \%$ \\
\hline $\mathbf{6}$ & Bayelsa & 463 & 336 & $73 \%$ & 297 & $64 \%$ & 186 & $40 \%$ & 158 & $34 \%$ \\
\hline $\mathbf{7}$ & Benue & 1243 & 676 & $54 \%$ & 613 & $49 \%$ & 522 & $42 \%$ & 572 & $46 \%$ \\
\hline $\mathbf{8}$ & Borno & 371 & 184 & $50 \%$ & 127 & $34 \%$ & 88 & $24 \%$ & 77 & $21 \%$ \\
\hline
\end{tabular}


A Survey of the Determinants of Effective E-Learning Education in Nigerian Secondary Schools:

A Way of Improving Economics Learning and Social Development in Post Covid-19

\begin{tabular}{|c|l|c|c|c|c|c|c|c|c|c|}
\hline $\mathbf{9}$ & Cross- & 760 & 447 & $59 \%$ & 361 & $48 \%$ & 482 & $63 \%$ & 482 & $63 \%$ \\
\hline $\mathbf{1 0}$ & Delta & 1169 & 882 & $75 \%$ & 739 & $63 \%$ & 738 & $63 \%$ & 598 & $51 \%$ \\
\hline $\mathbf{1 1}$ & Ebonyi & 601 & 344 & $57 \%$ & 390 & $65 \%$ & 288 & $48 \%$ & 306 & $51 \%$ \\
\hline $\mathbf{1 2}$ & Edo & 1022 & 708 & $69 \%$ & 559 & $55 \%$ & 524 & $51 \%$ & 472 & $46 \%$ \\
\hline $\mathbf{1 3}$ & Ekiti & 472 & 427 & $90 \%$ & 352 & $75 \%$ & 322 & $68 \%$ & 366 & $78 \%$ \\
\hline $\mathbf{1 4}$ & Enugu & 542 & 362 & $67 \%$ & 393 & $73 \%$ & 361 & $67 \%$ & 377 & $70 \%$ \\
\hline $\mathbf{1 5}$ & Fct-Abuja & 421 & 362 & $86 \%$ & 293 & $70 \%$ & 246 & $58 \%$ & 266 & $63 \%$ \\
\hline $\mathbf{1 6}$ & Gombe & 543 & 235 & $43 \%$ & 200 & $37 \%$ & 97 & $18 \%$ & 120 & $22 \%$ \\
\hline $\mathbf{1 7}$ & Imo & 688 & 412 & $60 \%$ & 378 & $55 \%$ & 389 & $57 \%$ & 427 & $62 \%$ \\
\hline $\mathbf{1 8}$ & Jigawa & 582 & 115 & $20 \%$ & 120 & $21 \%$ & 45 & $8 \%$ & 86 & $15 \%$ \\
\hline $\mathbf{1 9}$ & Kaduna & 912 & 672 & $74 \%$ & 550 & $60 \%$ & 517 & $57 \%$ & 481 & $53 \%$ \\
\hline $\mathbf{2 0}$ & Kano & 1684 & 1,171 & $70 \%$ & 859 & $51 \%$ & 653 & $39 \%$ & 703 & $42 \%$ \\
\hline $\mathbf{2 1}$ & Katsina & 550 & 331 & $60 \%$ & 245 & $45 \%$ & 266 & $48 \%$ & 282 & $51 \%$ \\
\hline $\mathbf{2 2}$ & Kebbi & 370 & 224 & $61 \%$ & 187 & $51 \%$ & 125 & $34 \%$ & 108 & $29 \%$ \\
\hline $\mathbf{2 3}$ & Kogi & 866 & 402 & $46 \%$ & 359 & $41 \%$ & 281 & $32 \%$ & 317 & $37 \%$ \\
\hline $\mathbf{2 4}$ & Kwara & 857 & 619 & $72 \%$ & 496 & $58 \%$ & 356 & $42 \%$ & 395 & $46 \%$ \\
\hline $\mathbf{2 5}$ & Lagos & 2830 & 2,764 & $98 \%$ & 2,445 & $86 \%$ & 1,951 & $69 \%$ & 1,971 & $70 \%$ \\
\hline $\mathbf{2 6}$ & Nasarawa & 896 & 584 & $65 \%$ & 507 & $57 \%$ & 387 & $43 \%$ & 369 & $41 \%$ \\
\hline $\mathbf{2 7}$ & Niger & 954 & 594 & $62 \%$ & 465 & $49 \%$ & 344 & $36 \%$ & 340 & $36 \%$ \\
\hline $\mathbf{2 8}$ & Ogun & 1376 & 1,332 & $97 \%$ & 1,249 & $91 \%$ & 1,013 & $74 \%$ & 1,073 & $78 \%$ \\
\hline $\mathbf{2 9}$ & Ondo & 665 & 568 & $85 \%$ & 434 & $65 \%$ & 419 & $63 \%$ & 462 & $69 \%$ \\
\hline $\mathbf{3 0}$ & Osun & 1047 & 746 & $71 \%$ & 583 & $56 \%$ & 429 & $41 \%$ & 526 & $50 \%$ \\
\hline $\mathbf{3 1}$ & Oyo & 1588 & 1,335 & $84 \%$ & 1,129 & $71 \%$ & 1,071 & $67 \%$ & 1,044 & $66 \%$ \\
\hline $\mathbf{3 2}$ & Plateau & 1069 & 746 & $70 \%$ & 693 & $65 \%$ & 570 & $53 \%$ & 508 & $48 \%$ \\
\hline $\mathbf{3 3}$ & Rivers & 1015 & 829 & $82 \%$ & 626 & $62 \%$ & 506 & $50 \%$ & 519 & $51 \%$ \\
\hline $\mathbf{3 4}$ & Sokoto & 344 & 184 & $53 \%$ & 193 & $56 \%$ & 120 & $35 \%$ & 135 & $39 \%$ \\
\hline $\mathbf{3 5}$ & Taraba & 632 & 283 & $45 \%$ & 259 & $41 \%$ & 203 & $32 \%$ & 186 & $29 \%$ \\
\hline $\mathbf{3 6}$ & Yobe & 213 & 108 & $51 \%$ & 94 & $44 \%$ & 42 & $20 \%$ & 45 & $21 \%$ \\
\hline $\mathbf{3 7}$ & Zamfara & 327 & 178 & $54 \%$ & 145 & $44 \%$ & 107 & $33 \%$ & 114 & $35 \%$ \\
\hline & & & & & & & & & & \\
\hline
\end{tabular}

The data on Table 1 revealed that Abia, Akwa-Ibom, Anambra, Delta, Ekiti, Enugu, FCT- Abuja, Imo, Kaduna, Lagos, Ogun, Ondo, Oyo and Rivers had more than 50\% e-learning facilities in their Secondary Schools. These imply that they will have effective online learning in various subjects including Economics during this Post Covid-19 era. Some States like Ebonyi, Edo, Kebbi, Nassarawa, Osun, Plateau and Sokoto had above 50\% in source of power supply, Computer and below $50 \%$ in laboratory and library. These results show that their inefficiency in laboratory and library will affect effective e-learning in this post Covid-19 era. The results also show that Adamawa, Bauchi, Bayelsa, Benue, Borno, Cross River, Ebonyi, Edo, Gombe, Jigawa, Kano, Kastina, Kogi, Niger, Taraba, Yobe and Zamfara had below 50\% elearning facilities in Secondary Schools. The implication is that these states will find it difficult to adopt e-learning education in post Covid-19 era.

Research Question 2: Analysis of percentage of Secondary Schools teachers' qualification for effective e-learning education? 
Table 2: Highest Teaching Qualification in Public and Private Secondary Schools by State and Gender

\begin{tabular}{|c|c|c|c|c|c|c|c|c|c|c|c|c|c|c|c|}
\hline & STATES & $\begin{array}{l}\mathrm{NOOF} \\
\mathrm{TRS}\end{array}$ & None & $\%$ & NCE & $\%$ & PGDE & $\%$ & $\begin{array}{l}\text { B.ED/ } \\
\text { BAED/ } \\
\text { BSC.ED }\end{array}$ & $\%$ & $\begin{array}{l}\text { MLED/ } \\
\text { MSCED/ } \\
\text { MAEDD }\end{array}$ & $\%$ & PHD & $\%$ & $\begin{array}{l}\% \text { of } \\
\text { QUA } \\
\text { TRS }\end{array}$ \\
\hline 1 & & 38 & & $.0 \%$ & 1,431 & $17.6 \%$ & 695 & $8.5 \%$ & 3,411 & $41.9 \%$ & 301 & $3.7 \%$ & 24 & $0.3 \%$ & $54.4 \%$ \\
\hline 2 & lamawa & 459 & 2,200 & $11.0 \%$ & 6,226 & $59.5 \%$ & 396 & $3.8 \%$ & 1,576 & $15.1 \%$ & 60 & $0.6 \%$ & 1 & $0.0 \%$ & $19.4 \%$ \\
\hline 3 & wa-Ihom & 9,675 & 303 & $4.1 \%$ & 1,377 & $14.2 \%$ & 1,346 & $13.9 \%$ & 3,459 & $35.8 \%$ & 181 & $1.9 \%$ & 9 & $0.1 \%$ & $51.6 \%$ \\
\hline 4 & ambra & 11,725 & 2,530 & $11.6 \%$ & 2,564 & $21.9 \%$ & 993 & $8.5 \%$ & 5,375 & $45.8 \%$ & 241 & $2.1 \%$ & 22 & $0.2 \%$ & $56.6 \%$ \\
\hline 5 & auchi & 2,872 & 2,373 & $18.4 \%$ & 7,882 & $61.2 \%$ & 391 & $3.0 \%$ & 2,136 & $16.6 \%$ & 89 & $0.7 \%$ & 1 & $0.0 \%$ & $20.3 \%$ \\
\hline 6 & yelsa & 4,093 & 1,348 & $32.9 \%$ & 671 & $16.4 \%$ & 298 & $7.3 \%$ & 1,714 & $41.9 \%$ & 60 & $1.5 \%$ & 2 & $0.0 \%$ & $50.7 \%$ \\
\hline 7 & Benue & 12,410 & 2,365 & $19.1 \%$ & 7,036 & $56.7 \%$ & 408 & $3.3 \%$ & 2,502 & $20.2 \%$ & 98 & $0.8 \%$ & 1 & $0.0 \%$ & $24.2 \%$ \\
\hline 8 & $\mathrm{mo}$ & 5,954 & 1,130 & $19.0 \%$ & 3,068 & $51.5 \%$ & 280 & $4.7 \%$ & 1,430 & $24.0 \%$ & 46 & $0.8 \%$ & 0 & $0.0 \%$ & $29.5 \%$ \\
\hline 9 & 0ss-River & 9,331 & 2,145 & $23.0 \%$ & 2,131 & $22.8 \%$ & 849 & $9.1 \%$ & 4,024 & $43.1 \%$ & 173 & $1.9 \%$ & 9 & $0.1 \%$ & $54.2 \%$ \\
\hline 10 & Delta & 14,488 & 3,730 & $25.7 \%$ & 3,178 & $21.9 \%$ & 950 & $6.6 \%$ & 6,392 & $44.1 \%$ & 228 & $1.6 \%$ & 10 & $0.1 \%$ & $52.3 \%$ \\
\hline 11 & Ebonyi & 6,425 & 1,324 & $20.6 \%$ & 2,764 & $43.0 \%$ & 261 & $4.1 \%$ & 1,989 & $31.0 \%$ & 82 & $1.3 \%$ & 5 & $0.1 \%$ & $36.4 \%$ \\
\hline 12 & $d d$ & 50 & 3,022 & $35.3 \%$ & 1,765 & $20.6 \%$ & 449 & $5.3 \%$ & 3,103 & $36.3 \%$ & 205 & $2.4 \%$ & 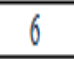 & $0.1 \%$ & $44.0 \%$ \\
\hline 13 & Ekith & 6,588 & 1,126 & $17.1 \%$ & 2,069 & $31.4 \%$ & 342 & $5.2 \%$ & 2,944 & $44.7 \%$ & 97 & $1.5 \%$ & 10 & $0.2 \%$ & $51.5 \%$ \\
\hline 14 & lugu & 340 & 1,464 & $15.7 \%$ & 3,629 & $38.9 \%$ & 768 & $8.2 \%$ & 3,314 & $35.5 \%$ & 160 & $1.7 \%$ & 5 & $0.1 \%$ & $45.5 \%$ \\
\hline 15 & dt-Abuja & 9,488 & 1,417 & $14.9 \%$ & 3,141 & $33.1 \%$ & 1,089 & $11.5 \%$ & 3,580 & $37.7 \%$ & 254 & $2.7 \%$ & 7 & $0.1 \%$ & $52.0 \%$ \\
\hline 16 & Gombe & 5,694 & 1,548 & $27.2 \%$ & 2,840 & $49.9 \%$ & 160 & $2.8 \%$ & 1,115 & $19.6 \%$ & 31 & $0.5 \%$ & 0 & $0.0 \%$ & $22.9 \%$ \\
\hline 17 & Imo & 10,123 & 1,811 & $17.9 \%$ & 1,149 & $11.4 \%$ & 1,007 & $9.9 \%$ & 5,819 & $57.5 \%$ & 319 & $3.2 \%$ & 18 & $0.2 \%$ & $70.8 \%$ \\
\hline 18 & Jigawa & 6,573 & 1,428 & $21.7 \%$ & 3,598 & $54.7 \%$ & 86 & $1.3 \%$ & 1,432 & $21.8 \%$ & 29 & $0.4 \%$ & 8 & $0.0 \%$ & $23.5 \%$ \\
\hline 19 & Kaduna & 10,268 & 2,599 & $25.3 \%$ & 3,707 & $36.1 \%$ & 881 & $8.6 \%$ & 2,948 & $28.7 \%$ & 129 & $1.3 \%$ & 4 & $0.0 \%$ & $38.6 \%$ \\
\hline 20 & Kano & 16,481 & 4,016 & $24.4 \%$ & 8,212 & $49.8 \%$ & 813 & $4.9 \%$ & 3,271 & $19.8 \%$ & 159 & $1.0 \%$ & 10 & $0.1 \%$ & $25.8 \%$ \\
\hline 21 & Katsina & 7,091 & 1,920 & $27.1 \%$ & 3,260 & $46.0 \%$ & 336 & $4.7 \%$ & 1,517 & $21.4 \%$ & 58 & $0.8 \%$ & 0 & $0.0 \%$ & $26.9 \%$ \\
\hline 22 & Kebbi & 5,271 & 1,586 & $30.1 \%$ & 2,086 & $39.6 \%$ & 289 & $5.5 \%$ & 1,282 & $24.3 \%$ & 28 & $0.5 \%$ & 0 & $0.0 \%$ & $30.3 \%$ \\
\hline 23 & $\mathrm{Kog}$ & 7,550 & 1,756 & $23.3 \%$ & 3,462 & $45.9 \%$ & 312 & $4.1 \%$ & 1,949 & $25.8 \%$ & 45 & $0.6 \%$ & 26 & $0.3 \%$ & $30.9 \%$ \\
\hline 24 & Krvara & 14,245 & 3,023 & $21.2 \%$ & 4,919 & $34.5 \%$ & 544 & $3.8 \%$ & 5,486 & $38.5 \%$ & 269 & $1.9 \%$ & 4 & $0.0 \%$ & $44.2 \%$ \\
\hline 25 & Lagos & 30,342 & 8,634 & $28.5 \%$ & 4,473 & $14.7 \%$ & 2,422 & $8.0 \%$ & 13,576 & $44.7 \%$ & 1,201 & $4.0 \%$ & 36 & $0.1 \%$ & $56.8 \%$ \\
\hline 26 & Nasarawa & 7,856 & 1,911 & $24.3 \%$ & 3,142 & $40.0 \%$ & 614 & $7.8 \%$ & 2,090 & $26.6 \%$ & 93 & $1.2 \%$ & 6 & $0.1 \%$ & $35.7 \%$ \\
\hline 27 & Niger & 9,999 & 2,621 & $26.2 \%$ & 4,143 & $41.4 \%$ & 497 & $5.0 \%$ & 2,639 & $26.4 \%$ & 98 & $1.0 \%$ & 1 & $0.0 \%$ & $32.4 \%$ \\
\hline 28 & Ogm. & 14,984 & 3,544 & $23.7 \%$ & 3,130 & $20.9 \%$ & 1,131 & $7.5 \%$ & 6,744 & $45.0 \%$ & 420 & $2.8 \%$ & 15 & $0.1 \%$ & $55.5 \%$ \\
\hline 29 & Ondo & 11,309 & 2,159 & $19.1 \%$ & 2,664 & $23.6 \%$ & 965 & $8.5 \%$ & 5,361 & $47.4 \%$ & 157 & $1.4 \%$ & 3 & $0.0 \%$ & $57.4 \%$ \\
\hline 30 & Osun & 13,144 & 3,568 & $27.1 \%$ & 3,437 & $26.1 \%$ & 901 & $6.9 \%$ & 5,059 & $38.5 \%$ & 174 & $1.3 \%$ & 5 & $0.0 \%$ & $46.7 \%$ \\
\hline 31 & Oyo & 19,946 & 3,819 & $19.1 \%$ & 5,686 & $28.5 \%$ & 1,045 & $5.2 \%$ & 8,887 & $44.6 \%$ & 499 & $2.5 \%$ & 10 & $0.1 \%$ & $52.3 \%$ \\
\hline 32 & Plateau & 12,008 & 2,397 & $20.0 \%$ & 6,313 & $52.6 \%$ & 581 & $4.8 \%$ & 2,518 & $21.0 \%$ & 194 & $1.6 \%$ & 5 & $0.0 \%$ & $27.5 \%$ \\
\hline 33 & Rivers & 20,357 & 5,891 & $28.9 \%$ & 1,854 & $9.1 \%$ & 2,435 & $12.0 \%$ & 9,580 & $47.1 \%$ & 570 & $2.8 \%$ & 27 & $0.1 \%$ & $62.0 \%$ \\
\hline 34 & Sokote & 5,206 & 1,251 & $24.0 \%$ & 2,221 & $42.7 \%$ & 261 & $5.0 \%$ & 1,419 & $27.3 \%$ & 54 & $1.0 \%$ & 0 & $0.0 \%$ & $33.3 \%$ \\
\hline 35 & Taraba & 7,000 & 1,825 & $26.1 \%$ & 3,376 & $48.2 \%$ & 231 & $3.3 \%$ & 1,522 & $21.7 \%$ & 46 & $0.7 \%$ & 0 & $0.0 \%$ & $25.7 \%$ \\
\hline 36 & Yobe & 3,140 & 679 & $21.6 \%$ & 1,391 & $44.3 \%$ & 147 & $4.7 \%$ & 905 & $28.8 \%$ & 17 & $0.5 \%$ & 1 & $0.0 \%$ & $34.1 \%$ \\
\hline 37 & Zamfara & 4,223 & 846 & $20.0 \%$ & 2,164 & $51.2 \%$ & 207 & $4.9 \%$ & 981 & $23.2 \%$ & 25 & $0.6 \%$ & 0 & $0.0 \%$ & $28.7 \%$ \\
\hline Total & & 82,346 & 90,585 & $23.7 \%$ & 126,159 & $33.0 \%$ & 25,380 & $6.6 \%$ & 133,049 & $34.8 \%$ & 6,890 & $1.8 \%$ & 283 & $0.1 \%$ & $43.3 \%$ \\
\hline
\end{tabular}


From the Table 2, the data indicate that Abia, Akwa-Ibom, Anambra, Bayelsa, Delta, Ekiti, FCT-Abuja, Imo, Lagos, Ogun, Ondo and Rivers had more than $50 \%$ of Secondary Schools teachers' qualification for effective e-learning education. The results also show that Imo had the highest (70\%) when compare to other states. Moreover, other states had below $50 \%$ of Secondary Schools teachers' qualification for effective e-learning education.

Research Question 3: Analysis of percentage of Secondary Schools teachers' that have computer literacy for effective elearning education?

Table 3: Number of Teaching Staff that are Computer Literate in Public and Private Secondary Schools by States and Level

\begin{tabular}{|c|l|c|c|c|}
\hline S/N & \multicolumn{1}{|c|}{ State } & $\begin{array}{c}\text { NO OF } \\
\text { TEACHERS }\end{array}$ & $\begin{array}{c}\text { Computer } \\
\text { Literate }\end{array}$ & $\begin{array}{c}\text { COMPUTER } \\
\text { LITERATE (\%) }\end{array}$ \\
\hline 1 & Abia & 8,138 & $\mathbf{6 , 1 1 2}$ & $75.1 \%$ \\
\hline 2 & Adamawa & 10,459 & $\mathbf{6 , 1 7 9}$ & $59.1 \%$ \\
\hline 3 & Akwa-Ibom & 9,675 & $\mathbf{8 , 1 6 9}$ & $84.4 \%$ \\
\hline 4 & Anambra & 11,725 & $\mathbf{9 , 9 8 3}$ & $85.1 \%$ \\
\hline 5 & Bauchi & 12,872 & $\mathbf{9 , 6 9 9}$ & $75.3 \%$ \\
\hline 6 & Bayelsa & 4,093 & $\mathbf{2 , 8 3 5}$ & $69.3 \%$ \\
\hline 7 & Benue & 12,410 & $\mathbf{7 , 9 4 3}$ & $64.0 \%$ \\
\hline 8 & Borno & 5,954 & $\mathbf{3 , 3 8 2}$ & $56.8 \%$ \\
\hline 9 & Cross-River & 9,331 & $\mathbf{7 , 1 5 2}$ & $76.6 \%$ \\
\hline 10 & Delta & 14,488 & $\mathbf{1 0 , 7 9 5}$ & $74.5 \%$ \\
\hline 11 & Ebonyi & 6,425 & $\mathbf{4 , 8 6 1}$ & $75.7 \%$ \\
\hline 12 & Edo & 8,550 & $\mathbf{6 , 0 9 2}$ & $71.3 \%$ \\
\hline 13 & Ekiti & 6,588 & $\mathbf{5 , 6 4 8}$ & $85.7 \%$ \\
\hline 14 & Enugu & 9,340 & $\mathbf{6 , 6 6 1}$ & $71.3 \%$ \\
\hline 15 & Fct-Abuja & 9,488 & $\mathbf{8 , 2 1 7}$ & $86.6 \%$ \\
\hline 16 & Gombe & 5,694 & $\mathbf{3 , 9 2 9}$ & $69.0 \%$ \\
\hline 17 & Imo & 10,123 & $\mathbf{8 , 4 9 5}$ & $83.9 \%$ \\
\hline 18 & Jigawa & 6,573 & $\mathbf{4 , 2 6 1}$ & $64.8 \%$ \\
\hline 19 & Kaduna & 10,268 & $\mathbf{8 , 1 9 1}$ & $79.8 \%$ \\
\hline 20 & Kano & 16,481 & $\mathbf{1 1 , 0 2 6}$ & $66.9 \%$ \\
\hline 21 & Katsina & 7,091 & $\mathbf{4 , 9 7 3}$ & $70.1 \%$ \\
\hline 22 & Kebbi & 5,271 & $\mathbf{3 , 3 8 3}$ & $64.2 \%$ \\
\hline 23 & Kogi & 7,550 & $\mathbf{5 , 8 0 1}$ & $76.8 \%$ \\
\hline 24 & Kwara & 19,245 & $\mathbf{1 1 , 2 2 6}$ & $78.8 \%$ \\
\hline 25 & Lagos & 9,342 & $\mathbf{2 6 , 5 9 8}$ & $87.7 \%$ \\
\hline 26 & Nasarawa & $\mathbf{5 , 3 7 7}$ & $68.4 \%$ \\
\hline 27 & Niger & 14,9894 & $\mathbf{1 2 , 7 4 3}$ & $85.0 \%$ \\
\hline 28 & Ogun & $\mathbf{8 , 9 9 6}$ & $79.5 \%$ \\
\hline 29 & Ondo & $\mathbf{1 1 , 3 7 2}$ & $86.5 \%$ \\
\hline 30 & Osun & & $86.6 \%$ \\
\hline 31 & Oyo & & \\
\hline & & & & \\
\hline
\end{tabular}




\begin{tabular}{|c|l|c|c|c|}
\hline 32 & Plateau & 12,008 & $\mathbf{8 , 0 0 5}$ & $66.7 \%$ \\
\hline 33 & Rivers & 20,357 & $\mathbf{1 7 , 3 1 6}$ & $85.1 \%$ \\
\hline 34 & Sokoto & 5,206 & $\mathbf{3 , 2 2 2}$ & $61.9 \%$ \\
\hline 35 & Taraba & 7,000 & $\mathbf{3 , 6 7 9}$ & $52.6 \%$ \\
\hline 36 & Yobe & 3,140 & $\mathbf{2 , 0 0 8}$ & $63.9 \%$ \\
\hline 37 & Zamfara & 4,223 & $\mathbf{2 , 7 8 3}$ & $65.9 \%$ \\
\hline & GRAND TOTAL & $\mathbf{3 8 2 , 3 4 6}$ & $\mathbf{2 9 1 , 1 3 3}$ & $\mathbf{7 6 . 1 \%}$ \\
\hline
\end{tabular}

Table 3 reveals that all the States had above 50\% of teaching staff that are computer literate in public and private Secondary Schools. This shows that most of the Secondary Schools teachers in private and public schools are computer literate. This implies that many teachers in private and public Secondary Schools underwent computer training; therefore, that is an indicator for effective e-learning education in post Covid-19 era.

\section{DISCUSSIONS}

The findings of this study revealed that some States such as Abia, Akwa-Ibom, Anambra, Delta, Ekiti, Enugu, FT-Abuja, Imo, Kaduna, Lagos, Ogun, Ondo, Oyo and Rivers had adequate provision of e-learning facilities (Source of power supply, computer, laboratory, library) in both private and public Secondary Schools for effective e-learning education while there are inadequate provision of e-learning facilities in other States. The findings further show that those States that lack elearning facilities tend to face the challenges of Covid-19 pandemic because they may find it difficult to adopt e-learning education in teaching and learning. These findings are in line with those of Eze, Chinedu-Eze and Bello (2018) who observed that availability and adequacy of e-learning facilities is very necessary for successful adoption of e-learning facilities in education system. The study is in consistent with Nwana (2012) who observed that there is shortage of elearning facilities in Nigerian Secondary Schools. Thus, availability of adequate e-learning facilities will improve the learning of Economics in Secondary Schools in this period of Covid-19 era.

Moreover, the findings also revealed that the percentage of Secondary Schools teachers' qualification is $43.3 \%$ for effective e-learning education which is low. The study also show that Imo State had the highest percentage of Secondary schools teachers' qualification for effective e-learning (70\%) followed by Rivers (62.2\%), Ondo (57.4\%), Lagos (56.8\%), Anambra (56.6\%), Ogun (55.5\%), Abia (54.4\%), Cross River (54.2\%), Delta (52.3\%), FCT- Abuja (52.0\%), Akwa-Ibom $(51.6 \%)$ and Ekiti $(51.5 \%)$ while other States have low percentage of qualifications. This implies that there is need for lecturers to upgrade their qualifications for effective delivery of instructions. This study corroborates with the findings of Nwagwu (2019) who discovered that excess workload is an obstacle to the performance of teachers because they may not have chance for further studies.

Results in table 3 showed that more than $50 \%$ of the Secondary Schools teachers in all the States are computer literate in public and private Schools. This study contradicts the findings of Mutua and Ng'eno (2016) who noted that lack of available and trained staff hinders effective e-learning in Secondary Schools. This study is also in disagreement with the findings of Eze, Chinedu-Eze and Bello (2018) who stated that teachers are insufficiently trained in Computer training. The findings of this study are in line with the findings of Mtebe and Raphael (2018) who observed that trained teachers in technology can provide reliable and effective help to students who use e-learning system. Furthermore, teachers may be 
computer literate but if there is insufficient provision of e-learning facilities it will affect effective e-learning adoption in Secondary Schools.

\section{CONCLUSIONS}

E-learning education is so important in education industry especially during this period of Covid-19 pandemic. Secondary School teachers and students are taking precautionary measures against Covid-19 disease. E-learning education will help in keeping social distance and still engage students even when the teachers are far away from the learners. E- Learning enhances Economics learning if the software is developed in such a way that it will be suitable and relevant to the subject. For effective e-learning in teaching and learning of Economics, there is need for the provision of adequate e-learning facilities such as source of power supply, computer devices, laboratory, and library, among others. The findings of this study revealed that not all the Secondary Schools in Nigeria have adequate provision of e-learning facilities and this will be a hindrance for effective e-learning education. Also, teachers' qualifications in secondary schools need to be improved to enable them to acquire more knowledge and skills for effective e-learning education. Although, most of the teachers in different states are computer literate but the question is on the issue of implementation. Again, insufficient provision of elearning facilities in some states will have a negative effect on effective e-learning education in Nigeria secondary schools during this period of post Covid-19 pandemic.

\section{Recommendations}

Based on the findings of this study, the following recommendations were made for effective e-learning education in Nigerian secondary schools:

- The government and principals of secondary schools should provide adequate e-learning facilities such as computers, internet, laboratory and library for effective e-learning.

- There is need to design e-learning curricula that is relevant with quality information suitable for each of the subjects. This will give the students opportunity to discuss their knowledge with other students or instructors outside their location.

- Principals should organize seminar for the teachers on technological pedagogy at least once in each session to improve their knowledge and skills.

\section{ACKNOWLEDGMENTS}

The researchers appreciate Dr Njideka Dorathy Eneogu who served as the corresponding author for this research. The office of the National Bureau of Statistics is also appreciated for making available the data for this research.

\section{REFERENCES}

1. Adelakein, I. (2020). Coronavirus (Covid-19) and Nigerian Education system: Impacts, Managemnt, Responses and way forward. Journal of Education, 88 - 102.

2. Amzat. J, Aminu. k, Kolo. V, Akinyele. A, Ogundairo. J, and Danjib. A. (2020). Coronavirus out break in Nigeria: Burden and socio-medical response during the first 100 days. International Journal of Infections Diseases, 98, 218 - 224. 
3. Arghya, R, Pradip, K.B \& Shilpee, A. D. (2020). Psychological analytics based technology adoption model for effective eductaional marketing in digital and social media marketing, emerging applications and theoretical developement. Switzerland: Springer.

4. Basak, S. K, Wotto, $M$ \& Belanger, P. (2017). Factors affecting to e-learning in continuing education in africa: a review of literature. International Journal of Engineering Sciences \& Management Research, 86 -97.

5. Eze, S. C, Chinedu-Eze \& Bello, A. O. (2018). The utilization of e-learning facilities in the educational delivery system of Nigeria: a study of M-University. International Journal of Educational Technology in Higher Education, 2 - 20.

6. Ihekweazu, C. (2020, September 16). Steps Nigeria is taking to prepare for cases of Coronavirus. Retrieved from http://the conversation.com/steps-nigeria-is-taking-to-prepare-for-cases-of-coronavirus-130704.

7. Khasawneh, M. (2015). Factors influence e-learning utilization in Jordanian universities-academic staff perspectives. Procedia - Social and Behavioural Sciences, 210, $170-180$.

8. Mtebe, J.S \& Raphael, C. (2018). Key factors in learners' satisfaction with the e-learning system at the university of Dar es Salaam, Tanzania. Australasian Journal of Educational technology, 107 - 122, Vol 34 (4).

9. Mutua, F. K \& Ng'eno, W. K. (2016). Determinants of e-learning in secondary schools in Kenya: a case of selected public secondary schools in Westlands district in Nairobi City County. International Journal of Humanities Social Sciences and Education, 3(9), 29 - 41.

10. Nations, U. (2020). Policy Brif: Education during Covid-19 and beyond. Retrieved from http://www.un.org.

11. NCDE. (2020). Covid-19 Nigeria. Retrieved from http://www.covid-19.ncdc.gov.ng.

12. Nwagwu, W. E. (2020). E-learning readiness of universities in Nigeria: what are the opinions of the academic staff of Nigeria's premier university. Journal of Education and Information Technologies, 25, 1343 - 1370.

13. Nwana, S. (2012). Challenges in the application of e-learning by secondary school teachers in Anambra State, Nigeria. Africa Journal of Teacher Education, 1-9.

14. Opoku, D\& Adu, I.N. (2016). Exploring the determinants of third generation (3G) mobile technology adoption among university students. Journal of Emerging Trends in Computing and Information Sciences, 7(6), 297 - 310.

15. Petit, D.D, Wharrad, O.H \& Windle, R. (2013). Exploring the underlying factors influencing e-learning adoption in nurse education. Journal of Advance Nursing, 1289 - 1300.

16. santos, W. G. (2020). Natural history of Covid-19 and current knowledge on treatment therapeutic options. Journal of Biomedicine and Pharmacotherapy, 129, 1 - 18.

17. Tornatzky, L\& Fleischer, M. (1990). The process of technology innovation. Lexington, MA: Lexington Books.

18. UNICEF. (2020, August 5th). Covid-19: Education transformed through remote learning. Retrieved from http://www.unicef.org/nigeria/stories.

19. Wanyaga, M, F, Kamau, J. W \& Gikandi, J. (2015). ICT Infrastructural factors that influence the adption of e-learning in public secondary schools in kenya. International Journal of Science and Research, 4 (6), 1061 - 1067.

20. WHO. (2020). Novel Coronavirus (2019- Cov) situation report. Retrieved from https:// www.worldmeters.info/.

21. Yakubu, M.N \& Dasuki, S. (2018). Assessing e-learning systems success in Nigeria: An application of the DeLone and McLean information systems success model. Journal of Information Technology Education Research, 183 -203. 